PROCEEDINGS OF THE

AMERICAN MATHEMATICAL SOCIETY

Volume 141, Number 1, January 2013, Pages 253-263

S 0002-9939(2012)11293-6

Article electronically published on May 9, 2012

\title{
QUASILINEAR ELLIPTIC EQUATIONS VIA PERTURBATION METHOD
}

\author{
XIANG-QING LIU, JIA-QUAN LIU, AND ZHI-QIANG WANG
}

(Communicated by Yingfei Yi)

\begin{abstract}
We present a new approach to studying a class of quasilinear problems including the so-called Modified Nonlinear Schrödinger Equations (MNLS). We show that solutions of quasilinear equations can be obtained as limits of 4-Laplacian perturbations.
\end{abstract}

\section{INTRODUCTION}

This paper is concerned with the existence and multiplicity of solutions for quasilinear equations of the form

$$
\left\{\begin{array}{l}
\sum_{i, j=1}^{N} D_{j}\left(a_{i j}(x, u) D_{i} u\right)-\frac{1}{2} \sum_{i, j=1}^{N} D_{s} a_{i j}(x, u) D_{i} u D_{j} u+f(x, u)=0, \text { in } \Omega, \\
u=0, \text { on } \partial \Omega,
\end{array}\right.
$$

where $D_{i}=\frac{\partial}{\partial x_{i}}$ and $D_{s} a_{i j}(x, s)=\frac{\partial}{\partial s} a_{i j}(x, s)$. This includes the following special class of equations (with $\left.a_{i j}(x, u)=\left(1+u^{2}\right) \delta_{i j}\right)$ :

$$
\Delta u+\frac{1}{2} u \Delta\left(u^{2}\right)+f(x, u)=0, \text { in } \Omega, u=0, \text { on } \partial \Omega,
$$

which is referred to as the so-called Modified Nonlinear Schrödinger Equation (MNLS). Here $\Omega \subset \mathbf{R}^{N}$ is a bounded smooth domain, and $f$ is a continuous function in $\bar{\Omega} \times \mathbf{R}$. This has been involved in models of mathematical physics, e.g., [3, 4, 5, 9, 10] and has received considerable attention in mathematical analysis in the last ten years.

In this paper we propose a new approach which works for the general form of the quasilinear equations. For the sake of simplicity of notation and of a clearer presentation of ideas we state and prove results for (MNLS) (1) in the first two sections, and mention and sketch generalizations in section 3. The weak form of the equation (1) is

$$
\int_{\Omega}\left[\left(1+u^{2}\right) \nabla u \nabla \phi+u|\nabla u|^{2} \phi-f(x, u) \phi\right] d x=0, \quad \forall \phi \in C_{0}^{\infty}(\Omega),
$$

which is formally the variational formulation of the following functional:

$$
I_{0}(u)=\frac{1}{2} \int_{\Omega}\left(1+u^{2}\right)|\nabla u|^{2} d x-\int_{\Omega} F(x, u) d x .
$$

Received by the editors January 26, 2011 and, in revised form, January 27, 2011; May 29, 2011; May 30, 2011; and June 9, 2011.

2010 Mathematics Subject Classification. Primary 35B05, 35B45.

(C)2012 American Mathematical Society Reverts to public domain 28 years from publication 
Here $F(x, s)=\int_{0}^{s} f(x, t) d t$, the primitive of $f$. We may define the derivative of $I_{0}$ at $u$ in the direction of $\phi \in C_{0}^{\infty}(\Omega)$ as follows:

$\left\langle I_{0}^{\prime}(u), \phi\right\rangle=\lim _{t \rightarrow 0+} \frac{1}{t}\left(I_{0}(u+t \phi)-I_{0}(u)\right)=\int_{\Omega}\left(\left(1+u^{2}\right) \nabla u \nabla \phi+u|\nabla u|^{2} \phi-f(x, u) \phi\right) d x$.

We call $u$ a critical point of $I_{0}$ if $u \in W_{0}^{1,2}(\Omega), \int_{\Omega} u^{2}|\nabla u|^{2} d x<\infty$ and $\left\langle I_{0}^{\prime}(u), \phi\right\rangle=0$ for all $\phi \in C_{0}^{\infty}(\Omega)$. That is, $u$ is a weak solution of (1).

For the nonlinearity we make the following assumptions.

$\left(f_{1}\right) \lim _{s \rightarrow 0} \frac{f(x, s)}{s}=0$ uniformly in $x$.

$\left(f_{2}\right)$ There exist $C_{1}>0,4<q<\frac{4 N}{N-2}$ such that for $x \in \Omega$ and $s \in \mathbf{R}$,

$$
|f(x, s)| \leq C_{1}\left(1+|s|^{q-1}\right) .
$$

$\left(f_{3}\right)$ There exist $M>0$ and $p>4$ such that for all $x \in \Omega,|s| \geq M, \frac{1}{p} s f(x, s) \geq$ $F(x, s)>0$.

$\left(f_{4}\right) f(x, s)$ is odd in $s$.

In general there is no suitable space in which $I_{0}$ enjoys both smoothness and compactness. There have been several ideas and approaches used in recent years to overcome the difficulties such as by minimizations [13, 15, the Nehari method [12, and change of variables [7, 11]. In this paper we propose a new approach. We consider a perturbed functional

$$
\begin{aligned}
I_{\mu}(u) & =\frac{1}{4} \mu \int_{\Omega}|\nabla u|^{4} d x+I_{0}(u) \\
& =\frac{1}{4} \mu \int_{\Omega}|\nabla u|^{4} d x+\frac{1}{2} \int_{\Omega}\left(1+u^{2}\right)|\nabla u|^{2} d x-\int_{\Omega} F(x, u) d x,
\end{aligned}
$$

where $\mu \in(0,1]$ is a parameter. Then it is easy to see that $I_{\mu}$ is a $C^{1}$-functional on $W_{0}^{1,4}(\Omega)$. For all $\phi \in W_{0}^{1,4}(\Omega)$,

(5) $\left\langle I_{\mu}^{\prime}(u), \phi\right\rangle=\mu \int_{\Omega}|\nabla u|^{2} \nabla u \nabla \phi d x+\int_{\Omega}\left(\left(1+u^{2}\right) \nabla u \nabla \phi+u|\nabla u|^{2} \phi-f(x, u) \phi\right) d x$.

The idea is to obtain existence of critical points of $I_{\mu}$ for $\mu>0$ small and to establish suitable estimates for the critical points as $\mu \rightarrow 0$ so that we may pass to the limit to get solutions of the original problem. This turns out to be effective for dealing with multiple solutions of quasilinear equations of general forms to which the idea of change of variables does not apply. Here are the main results of our paper for equation (11); see section 3 for results for more general quasilinear equations.

Theorem 1.1. Let $\mu_{n} \rightarrow 0$. Let $\left\{u_{n}\right\} \subset W_{0}^{1,4}(\Omega)$ be a sequence of critical points of $I_{\mu_{n}}$ satisfying $I_{\mu_{n}}^{\prime}\left(u_{n}\right)=0$ and $I_{\mu_{n}}\left(u_{n}\right) \leq C$ for some $C$ independent of $n$. Then up to a subsequence as $n \rightarrow \infty, u_{n} \rightarrow u$ in $W_{0}^{1,2}(\Omega), u_{n} \nabla u_{n} \rightarrow u \nabla u$ in $L^{2}(\Omega)$, $\mu_{n} \int_{\Omega}\left|\nabla u_{n}\right|^{4} d x \rightarrow 0$, and $I_{\mu_{n}}\left(u_{n}\right) \rightarrow I_{0}(u)$ and $u$ is a critical point of $I_{0}$.

Using Theorem 1.1 we have the following existence result.

Theorem 1.2. Assume $\left(f_{1}\right),\left(f_{2}\right),\left(f_{3}\right)$. Then

(i) $I_{\mu}$ has a positive and a negative critical point $u_{\mu}$ and $v_{\mu}$ and as $\mu \rightarrow 0, u_{\mu}$ (resp., $v_{\mu}$ ) converges to a positive (resp., negative) solution of equation (1).

(ii) If in addition $\left(f_{4}\right)$ holds, then $I_{\mu}$ has a sequence of critical points $u_{\mu, n}$ such that as $\mu \rightarrow 0, u_{\mu, n}$ converges to a solution $u_{n}$ of equation (11) with $I_{0}\left(u_{n}\right) \rightarrow \infty$ as $n \rightarrow \infty$.

Notation. We denote by $\|\cdot\|$ the norm of $W_{0}^{1,4}(\Omega)$ and by $|\cdot|_{s}$ the norm of $L^{s}(\Omega)(1 \leq$ $s<+\infty)$. 


\section{Proof of the MAIN RESUlts}

Lemma 2.1. For $\mu>0$ fixed, the functional $I_{\mu}$ satisfies the $(P S)$ condition.

Proof. Let $\left\{u_{n}\right\}$ be a (PS) sequence for $I_{\mu}$, i.e., $I_{\mu}\left(u_{n}\right) \rightarrow c$ and $I_{\mu}^{\prime}\left(u_{n}\right) \rightarrow 0$. We prove that $\left\{u_{n}\right\}$ has a convergent subsequence in $W_{0}^{1,4}(\Omega)$. First we have

$$
\begin{aligned}
I_{\mu}\left(u_{n}\right) & -\frac{1}{p}\left\langle I_{\mu}^{\prime}\left(u_{n}\right), u_{n}\right\rangle \\
= & \left(\frac{1}{4}-\frac{1}{p}\right) \mu \int_{\Omega}\left|\nabla u_{n}\right|^{4} d x+\left(\frac{1}{2}-\frac{1}{p}\right) \int_{\Omega}\left|\nabla u_{n}\right|^{2} d x \\
& +\left(\frac{1}{2}-\frac{2}{p}\right) \int_{\Omega} u_{n}^{2}\left|\nabla u_{n}\right|^{2} d x+\int_{\Omega}\left(\frac{1}{p} u_{n} f\left(x, u_{n}\right)-F\left(x, u_{n}\right)\right) d x .
\end{aligned}
$$

By $\left(f_{3}\right),\left(\frac{1}{4}-\frac{1}{p}\right) \mu \int_{\Omega}\left|\nabla u_{n}\right|^{4} d x \leq c+o\left(\| u_{n}||\right)$. This implies that $\left\{u_{n}\right\}$ is bounded in $W_{0}^{1,4}(\Omega)$. Up to a subsequence we may assume that $u_{n} \rightarrow u$ in $W_{0}^{1,4}(\Omega)$ and $u_{n} \rightarrow u$ in $L^{s}(\Omega)$ for $1 \leq s<\frac{4 N}{N-2}$. In (5) choosing $\phi=u_{n}-u_{m}$ we have

(7)

$$
\begin{aligned}
o(1) & || u_{n}-u_{m} \| \\
= & \left\langle I_{\mu}^{\prime}\left(u_{n}\right)-I_{\mu}^{\prime}\left(u_{m}\right), u_{n}-u_{m}\right\rangle \\
= & \mu \int_{\Omega}\left(\left|\nabla u_{n}\right|^{2} \nabla u_{n}-\left|\nabla u_{m}\right|^{2} \nabla u_{m}\right)\left(\nabla u_{n}-\nabla u_{m}\right) d x+\int_{\Omega}\left|\nabla u_{n}-\nabla u_{m}\right|^{2} d x \\
& +\int_{\Omega}\left(u_{n}^{2} \nabla u_{n}-u_{m}^{2} \nabla u_{m}\right)\left(\nabla u_{n}-\nabla u_{m}\right) d x \\
& +\int_{\Omega}\left(u_{n}\left|\nabla u_{n}\right|^{2}-u_{m}\left|\nabla u_{m}\right|^{2}\right)\left(u_{n}-u_{m}\right) d x \\
& -\int_{\Omega}\left(f\left(x, u_{n}\right)-f\left(x, u_{m}\right)\right)\left(u_{n}-u_{m}\right) d x .
\end{aligned}
$$

We may estimate the terms involved as follows:

$$
\begin{gathered}
\int_{\Omega}\left(u_{n}^{2} \nabla u_{n}-u_{m}^{2} \nabla u_{m}\right)\left(\nabla u_{n}-\nabla u_{m}\right) d x \\
=\int_{\Omega} u_{n}^{2}\left|\nabla u_{n}-\nabla u_{m}\right|^{2} d x+\int_{\Omega}\left(u_{n}^{2}-u_{m}^{2}\right) \nabla u_{m}\left(\nabla u_{n}-\nabla u_{m}\right) d x \\
\geq-\left|u_{n}-u_{m}\right|_{4}\left(\left|u_{n}\right|_{4}+\left|u_{m}\right|_{4}\right)|| u_{m}||\left(|| u_{n}||+|| u_{m}||\right) \rightarrow 0, \\
\left|\int_{\Omega}\left(u_{n}\left|\nabla u_{n}\right|^{2}-u_{m}\left|\nabla u_{m}\right|^{2}\right)\left(u_{n}-u_{m}\right) d x\right| \\
\leq\left(\left.\left|u_{n}\right|_{4}|| u_{n}\right|^{2}+\left|u_{m}\right|_{4}|| u_{m}||^{2}\right)\left|u_{n}-u_{m}\right|_{4} \rightarrow 0, \\
\left|\int_{\Omega}\left(f\left(x, u_{n}\right)-f\left(x, u_{m}\right)\right)\left(u_{n}-u_{m}\right) d x\right| \\
\leq C \int_{\Omega}\left(1+\left|u_{n}\right|^{q-1}+\left|u_{m}\right|^{q-1}\right)\left|u_{n}-u_{m}\right| d x \\
\leq C\left(1+\left|u_{n}\right|_{q}^{q-1}+\left|u_{m}\right|_{q}^{q-1}\right)\left|u_{n}-u_{m}\right|_{q} \rightarrow 0, \\
\mu \int_{\Omega}\left(\left|\nabla u_{n}\right|^{2} \nabla u_{n}-\left|\nabla u_{m}\right|^{2} \nabla u_{m}\right)\left(\nabla u_{n}-\nabla u_{m}\right) d x \geq c \mu \int_{\Omega}\left|\nabla u_{n}-\nabla u_{m}\right|^{4} d x .
\end{gathered}
$$

Returning to (7) we have

$$
c \mu \int_{\Omega}\left|\nabla u_{n}-\nabla u_{m}\right|^{4} d x \leq o(1)|| u_{n}-u_{m}||+o(1),
$$

which implies that $\left\|u_{n}-u_{m}\right\| \rightarrow 0$, i.e., $u_{n} \rightarrow u$ in $W_{0}^{1,4}(\Omega)$.

Next we consider the limiting behavior of critical points of $I_{\mu}$ as $\mu \rightarrow 0$ and prove Theorem 1.1 . 
Proof of Theorem 1.1. Similar to the proof of Lemma 2.1. by using $I_{\mu_{n}}^{\prime}\left(u_{n}\right)=0$ and $I_{\mu_{n}}\left(u_{n}\right) \leq C$ we obtain

$$
\begin{aligned}
C \geq & I_{\mu_{n}}\left(u_{n}\right)-\frac{1}{p}\left\langle I_{\mu_{n}}^{\prime}\left(u_{n}\right), u_{n}\right\rangle \\
= & \left(\frac{1}{4}-\frac{1}{p}\right) \mu_{n} \int_{\Omega}\left|\nabla u_{n}\right|^{4} d x+\left(\frac{1}{2}-\frac{1}{p}\right) \int_{\Omega}\left|\nabla u_{n}\right|^{2} d x+\left(\frac{1}{2}-\frac{2}{p}\right) \int_{\Omega} u_{n}^{2}\left|\nabla u_{n}\right|^{2} d x \\
& +\int_{\Omega}\left(\frac{1}{p} u_{n} f\left(x, u_{n}\right)-F\left(x, u_{n}\right)\right) d x .
\end{aligned}
$$

Thus by $\left(f_{3}\right)$,

$$
\mu_{n} \int_{\Omega}\left|\nabla u_{n}\right|^{4} d x+\int_{\Omega}\left|\nabla u_{n}\right|^{2} d x+\int_{\Omega} u_{n}^{2}\left|\nabla u_{n}\right|^{2} d x \leq C
$$

for some $C$ independent of $n$. Then up to a subsequence we have $u_{n} \rightarrow u$ in $W_{0}^{1,2}(\Omega), u_{n} \nabla u_{n} \rightarrow u \nabla u$ in $L^{2}(\Omega)$ and $u_{n}(x) \rightarrow u(x)$ a.e. $x \in \Omega$. Note that $u_{n}$ satisfies the equation

$$
\mu_{n} \int_{\Omega}\left|\nabla u_{n}\right|^{2} \nabla u_{n} \nabla \phi d x+\int_{\Omega}\left(\left(1+u_{n}^{2}\right) \nabla u_{n} \nabla \phi+u_{n}\left|\nabla u_{n}\right|^{2} \phi-f\left(x, u_{n}\right) \phi\right) d x=0,
$$

for all $\phi \in W_{0}^{1,4}(\Omega)$. Since $\left(\int_{\Omega}\left|u_{n}\right|^{\frac{4 N}{N-2}} d x\right)^{\frac{N-2}{N}} \leq c \int_{\Omega} u_{n}^{2}\left|D u_{n}\right|^{2} d x \leq c$, by Moser's iteration we have

$$
\left|u_{n}\right|_{L^{\infty}(\Omega)} \leq c ; \quad \text { hence }|u|_{L^{\infty}(\Omega)} \leq c
$$

for some constant $c$ independent of $n$. To show that $u$ is a critical point of $I_{0}$ we use some arguments in 6, 14 (see more references therein). In (14) we choose $\phi=\psi \exp \left(-u_{n}\right)$, where $\psi \in C_{0}^{\infty}(\Omega), \psi \geq 0$. Substituting $\phi$ into (14) we have

$$
\begin{aligned}
0= & \mu_{n} \int_{\Omega}\left|\nabla u_{n}\right|^{2} \nabla u_{n}\left(\nabla \psi \exp \left(-u_{n}\right)-\psi \nabla u_{n} \exp \left(-u_{n}\right)\right) d x \\
& +\int_{\Omega}\left(1+u_{n}^{2}\right) \nabla u_{n}\left(\nabla \psi \exp \left(-u_{n}\right)-\psi \nabla u_{n} \exp \left(-u_{n}\right)\right) d x \\
& +\int_{\Omega} u_{n}\left|\nabla u_{n}\right|^{2} \psi \exp \left(-u_{n}\right) d x-\int_{\Omega} f\left(x, u_{n}\right) \psi \exp \left(-u_{n}\right) d x \\
\leq & \mu_{n} \int_{\Omega}\left|\nabla u_{n}\right|^{2} \nabla u_{n} \nabla \psi \exp \left(-u_{n}\right) d x+\int_{\Omega}\left(1+u_{n}^{2}\right) \nabla u_{n} \nabla \psi \exp \left(-u_{n}\right) d x \\
& -\int_{\Omega}\left(1+u_{n}^{2}-u_{n}\right)\left|\nabla u_{n}\right|^{2} \psi \exp \left(-u_{n}\right) d x-\int_{\Omega} f\left(x, u_{n}\right) \psi \exp \left(-u_{n}\right) d x .
\end{aligned}
$$

Note that $1+u_{n}^{2}-u_{n} \geq 0$. By Fatou's lemma, the weak convergence of $u_{n}$ and the fact that $\mu_{n} \int_{\Omega}\left|\nabla u_{n}\right|^{4} d x$ is bounded, we have

$$
\begin{aligned}
0 \leq & \int_{\Omega}\left(1+u^{2}\right) \nabla u \nabla \psi \exp (-u) d x-\int_{\Omega}\left(1+u^{2}-u\right)|\nabla u|^{2} \psi \exp (-u) d x \\
& -\int_{\Omega} f(x, u) \psi \exp (-u) d x \\
= & \int_{\Omega}\left(1+u^{2}\right) \nabla u \nabla(\psi \exp (-u)) d x+\int_{\Omega} u|\nabla u|^{2} \psi \exp (-u) d x \\
& -\int_{\Omega} f(x, u) \psi \exp (-u) d x .
\end{aligned}
$$

Let $\chi \geq 0, \chi \in C_{0}^{\infty}(\Omega)$. We may choose a sequence of nonnegative functions $\psi_{n} \in C_{0}^{\infty}(\Omega)$ such that $\psi_{n} \rightarrow \chi \exp u$ in $W_{0}^{1,2}(\Omega), \psi_{n} \rightarrow \chi \exp u$ a.e. $x \in \Omega$, and $\psi_{n}$ is uniformly bounded in $L^{\infty}(\Omega)$. Then by approximations in (17) we may obtain for all $\chi \geq 0, \chi \in C_{0}^{\infty}(\Omega)$,

$$
\int_{\Omega}\left(1+u^{2}\right) \nabla u \nabla \chi d x+\int_{\Omega} u|\nabla u|^{2} \chi d x-\int_{\Omega} f(x, u) \chi d x \geq 0 .
$$

Similarly we may obtain an opposite inequality. Thus we have for all $\chi \in C_{0}^{\infty}(\Omega)$,

$$
\int_{\Omega}\left(1+u^{2}\right) \nabla u \nabla \chi d x+\int_{\Omega} u|\nabla u|^{2} \chi d x-\int_{\Omega} f(x, u) \chi d x=0 .
$$


That is, $u$ is a critical point of $I_{0}$ and a solution of equation (11). By doing approximations again we may have $u$ in the place of $\chi$ of (19):

$$
\int_{\Omega}\left(1+2 u^{2}\right)|\nabla u|^{2} d x-\int_{\Omega} f(x, u) u d x=0 .
$$

Setting $\phi=u_{n}$ in (14) we have

$$
\mu_{n} \int_{\Omega}\left|\nabla u_{n}\right|^{4} d x+\int_{\Omega}\left(1+2 u_{n}^{2}\right)\left|\nabla u_{n}\right|^{2} d x-\int_{\Omega} f\left(x, u_{n}\right) u_{n} d x=0 .
$$

Using $\int_{\Omega} f\left(x, u_{n}\right) u_{n} d x \rightarrow \int_{\Omega} f(x, u) u d x$, (20), (21) and lower semi-continuity we obtain

$$
\begin{gathered}
\int_{\Omega}\left|\nabla u_{n}\right|^{2} d x \rightarrow \int_{\Omega}|\nabla u|^{2} d x, \int_{\Omega} u_{n}^{2}\left|\nabla u_{n}\right|^{2} d x \rightarrow \int_{\Omega} u^{2}|\nabla u|^{2} d x, \\
\mu_{n} \int_{\Omega}\left|\nabla u_{n}\right|^{4} d x \rightarrow 0 .
\end{gathered}
$$

In particular, we have $u_{n} \rightarrow u$ in $W_{0}^{1,2}(\Omega), u_{n} \nabla u_{n} \rightarrow u \nabla u$ in $L^{2}(\Omega)$, and $I_{\mu_{n}}\left(u_{n}\right) \rightarrow$ $I_{0}(u)$.

Next we apply the Mountain Pass Theorem to obtain existence of critical points of $I_{\mu}$. For $\rho>0$ denote

$$
\Sigma_{\rho}=\left\{\left.u \in W_{0}^{1,4}(\Omega)\left|\int_{\Omega}\left(1+u^{2}\right)\right| \nabla u\right|^{2} d x \leq \rho^{2}\right\} .
$$

Consider a functional

$$
I_{\mu}^{+}(u)=\frac{1}{4} \mu \int_{\Omega}|\nabla u|^{4} d x+\frac{1}{2} \int_{\Omega}\left(1+u^{2}\right)|\nabla u|^{2} d x-\int_{\Omega} F\left(x, u^{+}\right) d x .
$$

Here and in the following we denote $u^{+}=\max \{u, 0\}$ and $u^{-}=\max \{-u, 0\}$. The functional $I_{\mu}$ satisfies the (PS) condition. Similarly we may verify that $I_{\mu}^{+}$satisfies the (PS) condition. By $\left(f_{1}\right)$ and $\left(f_{2}\right)$, for any $\epsilon>0$ there exists $C_{\epsilon}>0$ such that $F(x, u) \leq \epsilon u^{2}+C_{\epsilon}|u|^{q}$. Then for $\epsilon, \rho$ small,

$$
\begin{aligned}
\int_{\Omega} F\left(x, u^{+}\right) d x & \leq \int_{\Omega}\left(\epsilon u^{2}+C_{\epsilon}|u|^{q}\right) d x \\
& \leq \frac{1}{8} \int_{\Omega}|\nabla u|^{2} d x+C_{\epsilon}\left(\int_{\Omega} u^{2}|\nabla u|^{2} d x\right)^{q / 4} \\
& \leq \frac{1}{8} \rho^{2}+C_{\epsilon} \rho^{q / 2} \\
& \leq \frac{1}{4} \rho^{2} .
\end{aligned}
$$

Thus for small $\rho>0$ we have for $u \in \partial \Sigma_{\rho}$,

$$
I_{\mu}^{+}(u) \geq \frac{1}{2} \int_{\Omega}\left(1+u^{2}\right)|\nabla u|^{2} d x-\int_{\Omega} F\left(x, u^{+}\right) d x \geq \frac{1}{4} \rho^{2} .
$$

Choose $\phi \in C_{0}^{\infty}(\Omega), \phi(x) \geq 0$ in $\Omega$, and $T>0$. Define a path $h:[0,1] \rightarrow$ $W_{0}^{1,4}(\Omega)$ by $h(t)=t T \phi$. When $T$ is large enough we have $I_{\mu}^{+}(h(1))<0, \int_{\Omega}(1+$ $\left.h^{2}(1)\right)|\nabla h(1)|^{2} d x>\rho^{2}$ and $\sup _{t \in[0,1]} I_{\mu}^{+}(h(t)) \leq m$ for some $m$ independent of $\mu \in(0,1]$. Define

$$
c_{\mu}=\inf _{\gamma \in \Gamma} \sup _{t \in[0,1]} I_{\mu}^{+}(\gamma(t)),
$$

where

$$
\Gamma=\left\{\gamma \mid \gamma \in C\left([0,1], W_{0}^{1,4}(\Omega)\right), \gamma(0)=0, \gamma(1)=T \phi\right\} .
$$

From the Mountain Pass Theorem we have that $c_{\mu} \geq \frac{1}{4} \rho^{2}$ is a critical value of $I_{\mu}^{+}$. Let $u_{\mu}$ be a critical point corresponding to $c_{\mu}$. We have $u_{\mu} \geq 0$. Thus $u_{\mu}$ is a positive critical point of $I_{\mu}$. In summary we have 
Proposition 2.2. There exist positive constants $\rho$ and $m$ independent of $\mu$ such that $I_{\mu}$ has a positive critical point $u_{\mu}$ satisfying $\frac{1}{4} \rho^{2} \leq I_{\mu}\left(u_{\mu}\right) \leq m$.

Proof of Part (i) of Theorem 1.2. For a positive solution the proofs follow from Proposition 2.2 and Theorem 1.1. A similar argument gives a negative solution.

Next we turn to the symmetric case for which an unbounded sequence of critical values will be constructed. First we have the following.

Proposition 2.3. Assume $\left(f_{1,2,3,4}\right)$. Then for all $\mu \in(0,1]$ fixed, $I_{\mu}$ has a sequence of critical points $u_{\mu, j}$ such that there exist $\beta_{j}>\alpha_{j}$ both of which are independent of $\mu>0, \alpha_{j} \rightarrow \infty$ as $j \rightarrow \infty$ and $I_{\mu}\left(u_{\mu, j}\right) \in\left[\alpha_{j}, \beta_{j}\right]$ for all $\mu>0$.

Proof. Basically we apply the symmetric Mountain Pass Theorem due to Ambrosetti and Rabinowitz (see [2, 16]). In order to get uniform lower bounds of minimax critical values of $I_{\mu}$ in terms of $\mu>0$ we make some necessary changes. For completeness, we give the proof in detail.

Let $\lambda_{i}, i=1,2, \ldots$, be the $i$-th eigenvalue of $-\Delta$ with Dirichlet boundary condition and $\varphi_{i}$ the eigenfunction corresponding to $\lambda_{i}$. By regularity we know that $\phi_{i} \in W_{0}^{1,4}(\Omega)$. Let $E_{n}=\operatorname{span}\left\{\phi_{1}, \ldots, \phi_{n}\right\}$. We write $W_{0}^{1,4}(\Omega)=E_{j} \oplus X_{j}$ for $j=1,2, \ldots$, where $X_{j}$ is orthogonal to $E_{j}$ in $W_{0}^{1,2}(\Omega)$. Define

$$
G_{n}=\left\{H \in C\left(\Sigma_{R_{n}} \cap E_{n}, W_{0}^{1,4}(\Omega)\right): H \text { is odd and } H=i d \text { on } \partial \Sigma_{R_{n}} \cap E_{n}\right\},
$$

where $R_{n}>0$ is chosen such that $I_{\mu}(u)<0$ for $u \in E_{n} \backslash \Sigma_{R_{n}}$. Due to $\left(f_{3}\right)$, we may choose $R_{n}$ independent of $\mu>0$. Moreover, we assume $R_{n}>\rho_{n}$; see the following (24) for the definition of $\rho_{n}$. Set

$$
\begin{aligned}
\Gamma_{j}:= & \left\{H\left(\Sigma_{R_{n}} \cap E_{n} \backslash Y\right): H \in G_{n}, n \geq j, Y=-Y \subset \Sigma_{R_{n}} \cap E_{n}\right. \text { open, } \\
& \text { and } \gamma(Y) \leq n-j\},
\end{aligned}
$$

where $\gamma(\cdot)$ is the genus. Define

$$
c_{j}(\mu)=\inf _{B \in \Gamma_{j}} \sup _{u \in B} I_{\mu}(u), j=1,2, \ldots
$$

We claim that $c_{j}(\mu), j=1,2, \ldots$, are critical values of $I_{\mu}$ and there exist $\alpha_{j}<\beta_{j}$ such that $c_{j}(\mu) \in\left[\alpha_{j}, \beta_{j}\right]$ and $\alpha_{j} \rightarrow \infty$ as $j \rightarrow \infty$.

Since $I_{\mu}$ is increasing in $\mu$, we have $c_{j}(\mu) \leq c_{j}(1):=\beta_{j}, j=1,2, \ldots$. We estimate the lower bound for $c_{j}(\mu)$. By the following Lemma 2.4, we have an intersection property: If $\rho<R_{n}$ for all $n \geq j$, then

$$
\text { for } B \in \Gamma_{j} \text {, we have } B \cap \partial \Sigma_{\rho} \cap X_{j-1} \neq \emptyset \text {. }
$$

Hence

$$
c_{j}(\mu) \geq \inf _{u \in \partial \Sigma_{\rho} \cap X_{j-1}} I_{\mu}(u) \geq \inf _{u \in \partial \Sigma_{\rho} \cap X_{j-1}} I_{0}(u) .
$$

We may choose $\rho=\rho_{j}>0$ such that $\inf _{\partial \Sigma_{\rho_{j}} \cap X_{j-1}} I_{\mu}(u) \geq \alpha_{j}:=\frac{1}{8} \rho_{j}^{2} \rightarrow \infty$ as $j \rightarrow \infty$. In fact by $\left(f_{1}\right)$ and $\left(f_{2}\right)$, for any $\epsilon>0$ there exists $C=C_{\epsilon}>0$ such that $F(x, u) \leq \epsilon u^{2}+C|u|^{q}$. Then for $\epsilon$ small and $u \in \partial \Sigma_{\rho} \cap X_{j-1}$,

$$
\begin{aligned}
I_{\mu}(u) & \geq \frac{1}{2} \rho^{2}-\epsilon|u|_{2}^{2}-C|u|_{q}^{q} \\
& \geq \frac{1}{4} \rho^{2}-C|u|_{2 \cdot 2^{*}}^{a q}|u|_{2}^{(1-a) q} \\
& \geq \frac{1}{4} \rho^{2}-C \lambda_{j}^{-\frac{(1-a) q}{2}} \rho^{\frac{a q}{2}+(1-a) q} \\
& =\rho^{2}\left(\frac{1}{4}-C \lambda_{j}^{-\frac{(1-a) q}{2}} \rho^{\frac{a q}{2}+(1-a) q-2}\right) .
\end{aligned}
$$


Here $a \in(0,1)$ satisfies $\frac{1}{q}=\frac{a}{2 \cdot 2^{*}}+\frac{1-a}{2}$. As $\frac{a q}{2}+(1-a) q>\frac{q}{2}>2$ we may choose $\rho=\rho_{j}$ such that

$$
C \lambda_{j}^{-\frac{(1-a) q}{2}} \rho_{j}^{\frac{a q}{2}+(1-a) q-2}=\frac{1}{8} .
$$

Then $I_{\mu}(u) \geq \frac{1}{8} \rho_{j}^{2}$, and we have $\alpha_{j}=\frac{1}{8} \rho_{j}^{2} \rightarrow \infty$ as $j \rightarrow \infty$.

Now we show that $c_{j}(\mu), j=1,2, \ldots$, are critical values of $I_{\mu}$. If $\varphi: W_{0}^{1,4}(\Omega) \rightarrow$ $W_{0}^{1,4}(\Omega)$ is odd, and $\varphi=i d$ on $\partial \Sigma_{R_{n}} \cap E_{n}$ for all $n \geq j$, then $\varphi: \Gamma_{j} \rightarrow \Gamma_{j}$. Now $c_{j}(\mu) \geq \alpha_{j}>0$; hence $c_{j}(\mu)$ is a critical value of $I_{\mu}$. Otherwise there exist $\epsilon>0$ and $\varphi: W_{0}^{1,4}(\Omega) \rightarrow W_{0}^{1,4}(\Omega)$ such that $\varphi$ is odd, $\varphi\left(I_{\mu}^{c_{j}(\mu)+\epsilon}\right) \subset I_{\mu}^{c_{j}(\mu)-\epsilon}$ and $\varphi=i d$ in $I_{\mu}^{c_{j}(\mu)-\epsilon}$; in particular, $\varphi=i d$ on $\partial \Sigma_{R_{n}} \cap E_{n}$ for all $n$. By the definition of $c_{j}(\mu)$ there exists $B \in \Gamma_{j}$ such that $\sup _{u \in B} I_{\mu}(u) \leq c_{j}(\mu)+\epsilon$. Now $A=\varphi(B) \in \Gamma_{j}$ and $\sup _{u \in A} I_{\mu}(u) \leq c_{j}(\mu)-\epsilon$, which is a contradiction.

Lemma 2.4. For $B \in \Gamma_{j}$, it follows that $B \cap \partial \Sigma_{\rho} \cap X_{j-1} \neq \emptyset$ provided $\rho<R_{n}$ for all $n \geq j$.

Proof. Set $B=H\left(\Sigma_{R_{n}} \cap E_{n} \backslash Y\right)$ for some $n \geq j$ and $\gamma(Y) \leq n-j$. Let $\hat{O}=$ $\left\{x \in \Sigma_{R_{n}} \cap E_{n} \mid H(x) \in \Sigma_{\rho}\right\}$. Since $H$ is odd, $0 \in \hat{O}$. Let $O$ be the connected component of $\hat{O}$ containing 0 . Then $O$ is a bounded symmetric neighborhood of 0 in $E_{n}$. By Borsuk's theorem, $\gamma(\partial O)=n$. Since $H(u)=u$ for $u \in \partial \Sigma_{R_{n}} \cap E_{n}$, $\int_{\Omega}\left(1+H^{2}(u)\right)|D H(u)|^{2} d x=R_{n}^{2}>\rho^{2}$. Thus we have $H(u) \in \partial \Sigma_{\rho}$ for $u \in \partial O$. Set $W=\left\{u \in \Sigma_{R_{n}} \cap E_{n} \mid H(u) \in \partial \Sigma_{\rho}\right\}$. Then $\partial O \subset W$ and $\gamma(W)=n$. Also $\gamma(W \backslash Y) \geq n-(n-j)=j>j-1$. Then we have $\gamma(H(W \backslash Y))>j-1$. Since $\operatorname{codim} X_{j-1}=j-1, H(W \backslash Y) \cap X_{j-1} \neq \emptyset$. As $H(W \backslash Y) \subset B \cap \partial \Sigma_{\rho}$, the proof is finished.

Proof of Part (ii) of Theorem 1.2. For the existence of infinitely many solutions we proceed by using Proposition 2.3 and Theorem 1.1 and passing to the limit for $\mu \rightarrow 0$. As $\alpha_{j} \leq c_{j}(\mu) \leq \beta_{j}$ for all $\mu$ we may obtain critical point $u_{j}$ of $I_{0}$ with critical values in $\left[\alpha_{j}, \beta_{j}\right]$ and $I_{0}\left(u_{j}\right) \geq \alpha_{j} \rightarrow \infty$.

Remark 2.1. We note that the uniform lower bounds on the minimax values of $I_{\mu}$ involved always hold as long as there is $a_{0}>0$ such that $I_{\mu}(u) \geq \int_{\Omega}\left(\frac{a_{0}}{2}(1+\right.$ $\left.\left.u^{2}\right)|\nabla u|^{2}-F(x, u)\right) d x$ for $u \in W_{0}^{1,4}(\Omega)$. This is important for general quasilinear problems considered in the next section.

\section{General quasilinear Problems}

In this section we show that our new approach works for more general quasilinear equations. As the proofs are similar we will be sketchy from place to place. Consider (25)

$$
D_{j}\left(a_{i j}(x, u) D_{i} u\right)-\frac{1}{2} D_{s} a_{i j}(x, u) D_{i} u D_{j} u+f(x, u)=0, \text { in } \Omega, u=0 \text {, on } \partial \Omega .
$$

Here we use the conventional notation for summations. The functions $a_{i j}$ satisfy the following conditions.

(A1) $a_{i j} \in C^{1}(\bar{\Omega} \times \mathbf{R}, \mathbf{R}), a_{i j}=a_{j i}$, there exist constants $a_{1} \geq a_{0}>0$ such that

$$
a_{0}\left(1+s^{2}\right)|\xi|^{2} \leq a_{i j}(x, s) \xi_{i} \xi_{j} \leq a_{1}\left(1+s^{2}\right)|\xi|^{2}, \quad \forall x \in \bar{\Omega}, s \in \mathbf{R}, \xi \in \mathbf{R}^{N} .
$$

(A2) $0 \leq D_{s} a_{i j}(x, s) s \xi_{i} \xi_{j} \leq 2 a_{i j}(x, s) \xi_{i} \xi_{j}$ for $\xi \in \mathbf{R}^{N}, s \in \mathbf{R}$.

(A3) $a_{i j}(x, s)$ is odd in $s$. 
The variational functional now is

$$
I_{\mu}(u)=\frac{1}{4} \mu \int_{\Omega}|\nabla u|^{4} d x+\frac{1}{2} \int_{\Omega} a_{i j}(x, u) D_{i} u D_{j} u d x-\int_{\Omega} F(x, u) d x .
$$

Lemma 3.1. Assume $(A 1),(A 2),\left(f_{1}\right),\left(f_{2}\right),\left(f_{3}\right)$. For $\mu>0$ fixed, the functional $I_{\mu}$ satisfies the $(P S)$ condition.

Proof. Let $\left\{u_{n}\right\}$ be a (PS) sequence for $I_{\mu}$, i.e., $I_{\mu}\left(u_{n}\right) \rightarrow c$ and $I_{\mu}^{\prime}\left(u_{n}\right) \rightarrow 0$. We prove that $\left\{u_{n}\right\}$ has a convergent subsequence in $W_{0}^{1,4}(\Omega)$. First we have

$$
\begin{aligned}
& I_{\mu}\left(u_{n}\right)-\frac{1}{p}\left\langle I_{\mu}^{\prime}\left(u_{n}\right), u_{n}\right\rangle \\
& \quad=\left(\frac{1}{4}-\frac{1}{p}\right) \mu \int_{\Omega}\left|\nabla u_{n}\right|^{4} d x+\left(\frac{1}{2}-\frac{1}{p}\right) \int_{\Omega} a_{i j}\left(x, u_{n}\right) D_{i} u_{n} D_{j} u_{n} d x \\
& \quad-\frac{1}{2 p} \int_{\Omega} D_{s} a_{i j}\left(x, u_{n}\right) u_{n} D_{i} u_{n} D_{j} u_{n} d x+\int_{\Omega}\left(\frac{1}{p} u_{n} f\left(x, u_{n}\right)-F\left(x, u_{n}\right)\right) d x .
\end{aligned}
$$

By $\left(f_{4}\right)$ and $(A 1)-(A 2),\left(\frac{1}{4}-\frac{1}{p}\right) \mu \int_{\Omega}\left|\nabla u_{n}\right|^{4} d x \leq c+o\left(\left\|u_{n}\right\|\right)$. This implies that $\left\{u_{n}\right\}$ is bounded in $W_{0}^{1,4}(\Omega)$. Up to a subsequence we may assume $u_{n} \rightarrow u$ in $W_{0}^{1,4}(\Omega)$ and $u_{n} \rightarrow u$ in $L^{s}(\Omega)$ for $1 \leq s<\frac{4 N}{N-2}$. In (5) choosing $\phi=u_{n}-u_{m}$ we have

$$
\begin{aligned}
o(1) & \left\|u_{n}-u_{m}\right\| \\
= & \left\langle I_{\mu}^{\prime}\left(u_{n}\right)-I_{\mu}^{\prime}\left(u_{m}\right), u_{n}-u_{m}\right\rangle \\
= & \mu \int_{\Omega}\left(\left|\nabla u_{n}\right|^{2} \nabla u_{n}-\left|\nabla u_{m}\right|^{2} \nabla u_{m}\right)\left(\nabla u_{n}-\nabla u_{m}\right) d x \\
& +\int_{\Omega}\left(a_{i j}\left(x, u_{n}\right) D_{i} u_{n}-a_{i j}\left(x, u_{m}\right) D_{i} u_{m}\right)\left(D_{j} u_{n}-D_{j} u_{m}\right) d x \\
& +\int_{\Omega} \frac{1}{2}\left(D_{s} a_{i j}\left(x, u_{n}\right) D_{i} u_{n} D_{j} u_{n}-D_{s} a_{i j}\left(x, u_{m}\right) D_{i} u_{m} D_{j} u_{m}\right)\left(u_{n}-u_{m}\right) d x \\
& -\int_{\Omega}\left(f\left(x, u_{n}\right)-f\left(x, u_{m}\right)\right)\left(u_{n}-u_{m}\right) d x .
\end{aligned}
$$

Using $(A 1)-(A 2)$ we show that the limits of all terms except the first are nonnegative and we obtain

$$
c \mu \int_{\Omega}\left|\nabla u_{n}-\nabla u_{m}\right|^{4} d x \leq o(1)|| u_{n}-u_{m} \|+o(1),
$$

which implies $\left\|u_{n}-u_{m}\right\| \rightarrow 0$, i.e., $u_{n} \rightarrow u$ in $W_{0}^{1,4}(\Omega)$.

Next we show that Theorem 1.1 still holds in this general situation. We assume $(A 1),(A 2),\left(f_{1}\right),\left(f_{2}\right),\left(f_{3}\right)$.

Proof. Similar to the proof of the above lemma, by using $I_{\mu_{n}}^{\prime}\left(u_{n}\right)=0$ and $I_{\mu_{n}}\left(u_{n}\right) \leq$ $C$,

$$
\mu_{n} \int_{\Omega}\left|\nabla u_{n}\right|^{4} d x+\int_{\Omega} a_{i j}\left(x, u_{n}\right) D_{i} u_{n} D_{j} u_{n} d x \leq C
$$

for some $C$ independent of $n$. Then by $(A 1)$ up to a subsequence we have $u_{n} \rightarrow u$ in $W_{0}^{1,2}(\Omega), u_{n} \nabla u_{n} \rightarrow u \nabla u$ in $L^{2}(\Omega)$ and $u_{n}(x) \rightarrow u(x)$ a.e. $x \in \Omega$. Note that $u_{n}$ satisfies, for all $\phi \in W_{0}^{1,4}(\Omega)$,

$$
\begin{aligned}
& \mu_{n} \int_{\Omega}\left|\nabla u_{n}\right|^{2} \nabla u_{n} \nabla \phi d x+\int_{\Omega} a_{i j}\left(x, u_{n}\right) D_{i} u_{n} D_{j} \phi d x \\
& \left.\quad+\int_{\Omega} \frac{1}{2} D_{s} a_{i j}\left(x, u_{n}\right) D_{i} u_{n} D_{j} u_{n} \phi d x-\int_{\Omega} f\left(x, u_{n}\right) \phi\right) d x=0 .
\end{aligned}
$$


Since $\left(\int_{\Omega}\left|u_{n}\right|^{\frac{4 N}{N-2}} d x\right)^{\frac{N-2}{N}} \leq c \int_{\Omega} a_{i j}\left(x, u_{n}\right) D_{i} u_{n} D_{j} u_{n} d x \leq c$, by Morser's iteration we have

$$
\left|u_{n}\right|_{L^{\infty}(\Omega)} \leq c ; \quad \text { hence }|u|_{L^{\infty}(\Omega)} \leq c
$$

for some $c$ independent of $n$. We use the same argument as before to show that $u$ is a critical point of $I_{0}$. In (28) we choose $\phi=\psi \exp \left(-M u_{n}\right)$, where $\psi \geq 0, \psi \in C_{0}^{\infty}(\Omega)$, and $M>0$ is a constant. Substituting $\phi$ into (28) we have

$$
\begin{aligned}
0= & \mu_{n} \int_{\Omega}\left|\nabla u_{n}\right|^{2} \nabla u_{n}\left(\nabla \psi \exp \left(-M u_{n}\right)-M \psi \nabla u_{n} \exp \left(-M u_{n}\right)\right) d x \\
& +\int_{\Omega} a_{i j}\left(x, u_{n}\right) D_{i} u_{n}\left(D_{j} \psi \exp \left(-M u_{n}\right)-M \psi D_{j} u_{n} \exp \left(-M u_{n}\right)\right) d x \\
& +\int_{\Omega} \frac{1}{2} D_{s} a_{i j}\left(x, u_{n}\right) D_{i} u_{n} D_{j} u_{n} \psi \exp \left(-M u_{n}\right) d x \\
& -\int_{\Omega} f\left(x, u_{n}\right) \psi \exp \left(-M u_{n}\right) d x \\
\leq & \mu_{n} \int_{\Omega}\left|\nabla u_{n}\right|^{2} \nabla u_{n} \nabla \psi \exp \left(-M u_{n}\right) d x \\
& +\int_{\Omega} a_{i j}\left(x, u_{n}\right) D_{i} u_{n} D_{j} \psi \exp \left(-M u_{n}\right) d x \\
& -\int_{\Omega}\left(M a_{i j}\left(x, u_{n}\right)-\frac{1}{2} D_{s} a_{i j}\left(x, u_{n}\right)\right) D_{i} u_{n} D_{j} u_{n} \psi \exp \left(-M u_{n}\right) d x \\
& -\int_{\Omega} f\left(x, u_{n}\right) \psi \exp \left(-M u_{n}\right) d x .
\end{aligned}
$$

Note that $M a_{i j}\left(x, u_{n}\right)-\frac{1}{2} D_{s} a_{i j}\left(x, u_{n}\right)$ is positive for $M$ large enough. By Fatou's lemma, the weak convergence of $u_{n}$ and the fact that $\mu_{n} \int_{\Omega}\left|\nabla u_{n}\right|^{4} d x$ is bounded, we have

$$
\begin{aligned}
0 \leq & \int_{\Omega} a_{i j}(x, u) D_{i} u D_{j} \psi \exp (-M u) d x \\
& -\int_{\Omega}\left(M a_{i j}(x, u)-\frac{1}{2} D_{s} a_{i j}(x, u)\right) D_{i} u D_{j} u \psi \exp (-M u) d x \\
& -\int_{\Omega} f(x, u) \psi \exp (-M u) d x \\
= & \int_{\Omega} a_{i j}(x, u) D_{i} u D_{j}(\psi \exp (-M u)) d x \\
& +\frac{1}{2} \int_{\Omega} D_{s} a_{i j}(x, u) D_{i} u D_{j} u \psi \exp (-M u) d x-\int_{\Omega} f(x, u) \psi \exp (-M u) d x .
\end{aligned}
$$

From here the same arguments used before give for all $\chi \in C_{0}^{\infty}(\Omega)$,

$$
\int_{\Omega} a_{i j}(x, u) D_{i} u D_{j} \chi d x+\frac{1}{2} \int_{\Omega} D_{s} a_{i j}(x, u) D_{i} u D_{j} u \chi d x-\int_{\Omega} f(x, u) \chi d x=0 .
$$

That is, $u$ is a critical point of $I_{0}$ and a solution of equation (25). By doing approximations again we may have $u$ in the place of $\chi$ of (32):

$$
\int_{\Omega} a_{i j}(x, u) D_{i} u D_{j} u d x+\frac{1}{2} \int_{\Omega} D_{s} a_{i j}(x, u) u D_{i} u D_{j} u d x-\int_{\Omega} f(x, u) u d x=0 .
$$

Setting $\phi=u_{n}$ in (28) we have

$$
\begin{aligned}
& \mu_{n} \int_{\Omega}\left|\nabla u_{n}\right|^{4} d x+\int_{\Omega} a_{i j}\left(x, u_{u}\right) D_{i} u_{n} D_{j} u_{n} d x+\frac{1}{2} \int_{\Omega} D_{s} a_{i j}\left(x, u_{n}\right) u_{n} D_{i} u_{n} D_{j} u_{n} d x \\
& \quad-\int_{\Omega} f\left(x, u_{n}\right) u_{n} d x=0 .
\end{aligned}
$$

Using $\int_{\Omega} f\left(x, u_{n}\right) u_{n} d x \rightarrow \int_{\Omega} f(x, u) u d x$, (33), (34) and lower semi-continuity we obtain

$$
\mu_{n} \int_{\Omega}\left|\nabla u_{n}\right|^{4} d x \rightarrow 0, \int_{\Omega} a_{i j}\left(x, u_{n}\right) D_{i} u_{n} D_{j} u_{n} d x \rightarrow \int_{\Omega} a_{i j}(x, u) D_{i} u D_{j} u d x
$$

which implies that $u_{n} \rightarrow u$ in $W_{0}^{1,2}(\Omega), u_{n} \nabla u_{n} \rightarrow u \nabla u$ in $L^{2}(\Omega)$, and $I_{\mu_{n}}\left(u_{n}\right) \rightarrow$ $I_{0}(u)$. 
Using this convergence theorem we can obtain the following existence result for equation (25).

Theorem 3.2. Assume $(A 1),(A 2),\left(f_{1}\right),\left(f_{2}\right),\left(f_{3}\right)$. Then

(i) $I_{\mu}$ has a positive and a negative critical point $u_{\mu}$ and $v_{\mu}$ and as $\mu \rightarrow 0, u_{\mu}$ (resp., $v_{\mu}$ ) converges to a positive (resp., negative) solution of equation (25).

(ii) If in addition $\left(A_{3}\right),\left(f_{4}\right)$ hold, then $I_{\mu}$ has a sequence of critical points $u_{\mu, n}$ such that as $\mu \rightarrow 0, u_{\mu, n}$ converges to a solution $u_{n}$ of equation (25) with $I_{0}\left(u_{n}\right) \rightarrow$ $\infty$ as $n \rightarrow \infty$.

The proof is similar to the proof of Theorem 1.2 with a few modifications. First, by using the Mountain Pass Theorem we obtain a positive critical point of $I_{\mu}$, and the convergence theorem gives a positive critical point of $I_{0}$. Second, by the symmetric Mountain Pass Theorem we obtain a sequence of unbounded critical values of $I_{\mu}$ with uniform lower and upper bounds (see Remark 2.1). Then the convergence theorem gives solutions for $I_{0}$. The details are omitted.

We close the paper by pointing out that to our knowledge there have not been any multiplicity results for general quasilinear equations such as (25) and our results above are the first attempt in this direction, though for the special form of equations (11), multiplicity results could be derived by using the change of variable arguments from [7, 11] (we refer to recent work [1, 8, 17, for more references on using the arguments of changing variables for various problems).

\section{ACKNOWLEDGMENTS}

The authors would like to thank the referee for a thoughtful reading of an earlier version of the paper, which encouraged the authors to give a better presentation of their results in the later version. The research of the first and the second authors is supported by TianYuan Special Funds of NSFC (11026210) and the NSFC (10871110) respectively.

\section{REFERENCES}

[1] C. O. Alves, G. M. Figueiredo and U.B. Severo, Multiplicity of positive solutions for a class of quasilinear problems, Adv. Differential Equations, 14 (2009), 911-942. MR2548282 (2011a:35145)

[2] A. Ambrosetti and P. Rabinowitz, Dual variational methods in critical point theory and applications, J. Funct. Anal., 14 (1973), 349-381. MR0370183(51:6412)

[3] Y. Brihaye and B. Hartmann, Solitons on nanotubes and fullerenes as solutions of a modified nonlinear Schrödinger equation, Advances in soliton research, 135-151, Nova Sci. Publ., Hauppauge, NY, 2006. MR.2215050

[4] L. Brizhik, A. Eremko, B. Piette and W.J. Zakrzewski, Static solutions of a D-dimensional modified nonlinear Schrödinger equation, Nonlinearity, 16 (2003), 1481-1497. MR.1986307 (2004e:35206)

[5] L. Brüll and H. Lange, Solitary waves for quasilinear Schrödinger equations, Expos. Math., 4 (1986), 278-288. MR880128 (88i:35145)

[6] A. Canino and M. Degiovanni, Nonsmooth critical point theory and quasilinear elliptic equations, Topol. methods in differential equations and inclusions (Montreal, PQ, 1994), 1-50, NATO Adv. Sci. Inst. Ser. C Math. Phys. Sci., 472, 1995. MR1368669 (96m:58036)

[7] M. Colin and L. Jeanjean, Solutions for a quasilinear Schrödinger equation: a dual approach, Nonlinear Anal., 56 (2004), 213-226. MR2029068 (2004k:35110)

[8] J.M. do Ó, O. Miyagaki and S. Soares, Soliton solutions for quasilinear Schrödinger equations with critical growth, J. Differential Equations, 248 (2010), 722-744. MR2578446 (2011b:35489) 
[9] B. Hartmann and W. J. Zakrzewski, Electrons on hexagonal lattices and applications to nanotubes, Phys. Rev. B, 68 (2003), 184-302.

[10] S. Kurihura, Large-amplitude quasi-solitons in superfluid films, J. Phys. Soc. Japan, 50 (1981), 3262-3267.

[11] J.-Q. Liu, Y. Wang and Z.-Q. Wang, Soliton solutions for quasilinear Schrödinger equations, II. J. Differential Equations, 187 (2003), 473-493. MR1949452 (2004e:35074)

[12] J.-Q. Liu, Y. Wang and Z.-Q. Wang, Solutions for quasilinear Schrödinger equations via the Nehari method, Comm. in Partial Differential Equations, 29 (2004), 879-901. MR2059151 (2005d:35080)

[13] J.-Q. Liu and Z.-Q. Wang, Soliton solutions for quasilinear Schrödinger equations. I, Proc. Amer. Math. Soc., 131 (2003), 441-448. MR1933335 (2003k:35232)

[14] J.-Q. Liu, Z.-Q. Wang, Bifurcations for quasilinear Schrödinger equations, II. Comm. in Contemporary Mathematics, 10 (2008), 723-743. MR.2446896 (2009m:35146)

[15] M. Poppenburg, K. Schmitt and Z.-Q. Wang, On the existence of solutions to quasilinear Schrödinger equations, Calculus of Variations and PDEs, 14 (2002), 329-344. MR1899450 (2003d:35247)

[16] P.H. Rabinowitz, Minimax methods in critical point theory with applications to differential equations, CBMS Reg. Conf. Ser. Math. 65, Amer. Math. Soc., Providence, RI, 1986. MR845785 (87j:58024)

[17] E.A.B. Silva and G.F. Vieira, Quasilinear asymptotically periodic Schrödinger equations with critical growth, Calc. Var. Partial Differential Equations 39 (2010), 1-33. MR2659677 (2011f:35140)

Department of Mathematics, Yunnan Normal University, Kunming, 650092, People's Republic of China

E-mail address: 1xq8u8@163.com

Department of Mathematics, Peking University, Beijing, 100871, People's Republic OF CHINA

E-mail address: jiaquan@math.pku.edu.cn

Chern Institute of Mathematics, Nankai University, Tianjin, 300071, People's Republic of China - and - Department of Mathematics and Statistics, Utah State UniVERSITY, LOGAN, UTAH 84322

E-mail address: zhi-qiang.wang@usu.edu 